\title{
Editorial series: cancer care in low- and middle-income countries
}

\author{
Jörg Haier ${ }^{1,4}$. Jonathan Sleeman ${ }^{2,3}$. Jürgen Schäfers ${ }^{4}$
}

Published online: 31 October 2019

(c) Springer Nature B.V. 2019

Universal health coverage (UHC) is a central goal for healthcare systems worldwide. When evaluating progress towards the implementation of UHC in a given country, the World Health Organization (WHO) considers three dimensions of policy evaluation: the population groups within the country that are covered, the extent of financial protection for access to healthcare services, and the spectrum of services that are available. Although health policy agendas have helped to improve the quality of care in many low- and middle-income countries (LMICs), including the Eastern Mediterranean Region, sub-Saharan Africa and East Asia, progress to date in achieving UHC has not been optimal. Some of these countries with large populations, such as India and Indonesia, are currently implementing or rolling-out health insurance schemes that aim to cover the entire population in order to meet UHC criteria. However, adequate access to primary care as a key requirement of UHC is not universally achieved in many countries, particularly for vulnerable populations.

Cancer care represents a major hurdle for UHC in LMICs, mainly due to lack of screening and early detection, poor education with regard to cancer, the cultural taboo of cancer, and the requirement for costly infrastructure for diagnosis and treatment, including educated personnel. This results in an unnecessarily high proportion of patients with metastatic and advanced tumor stages. In this editorial we outline how intentional and non-intentional consequences of policy decisions as well as the implementation of incentives during

Jörg Haier

haier.joerg@mh-hannover.de

1 Comprehensive Cancer Center, Hannover Medical School, Hanover, Germany

2 Medical Faculty Mannheim, University of Heidelberg, CBTM, Ludolf-Krehl-Str. 13 - 17, 68167 Mannheim, Germany

3 Karlsruhe Institute for Technology (KIT), Campus Nord, Institut für Toxikologie und Genetik, Postfach 3640, 76021 Karlsruhe, Germany

4 IGP-Institute for Health Sciences and Public Health, Muenster, Germany progress towards UHC can have a major impact on the quality and scope of cancer care in these countries.

\section{Deficits in healthcare policy and incentives, and their impact on cancer care in LMICs}

For many LMICs, significant differences between regions, between rural and urban populations, and across social groups remain major challenges for the reorganization of existing healthcare delivery, and the implementation of new structures that enable basic equal access to healthcare for the entire population. A key deficiency is often the absence or insufficient implementation of an explicit national policy for quality improvement and patient safety in cancer care as part of the national health system. In particular, the incentivizing effects of legal measures, national plans, and implementation concepts are poorly understood, resulting in unforeseen consequences that frequently result in limited benefits for cancer patients. In addition, incentive systems that link contractual agreement, regulations, accreditation, and performance indicators are underutilized or absent in most LMIC countries.

Insufficient cancer care coverage in LIMCs is to a large extent caused by high out-of-pocket healthcare expenditures (accessibility) and by the adverse consequences of poor quality of care. In addition, rural and remote areas frequently suffer from insufficient healthcare infrastructure, and even more from a lack of qualified healthcare personnel (availability). In cancer care this results in insufficient implementation of preventive treatments, a lack of early diagnosis, and subsequently a high incidence of insufficient treatment, especially in the context of advanced metastatic disease. A further barrier to achieving UHC using healthcare insurance schemes that are available to the entire population is the implementation of incentives that efficiently guide and motivate both cancer care providers and cancer patients in an appropriate manner to use existing healthcare structures and funds as effectively and efficiently as possible. If these incentives are not set properly, financial and human resources will be wasted, and the provision of quality 
care will not be stimulated. Improper incentives may also inappropriately influence the development of cancer care systems due to conflicts of interests (CoI) and unreasonable prioritization, amongst other factors. Incentives are powerful tools that can help shape a political cancer care framework. However, their effects on healthcare development is largely independent of political targets, the use of feedback mechanisms such as key performance indicators, and the amount of available healthcare funds. Thus to overcome the impaired availability of cancer care delivery structures in LIMCs and thereby improve UHC, the guidance function of key factors such as economic and structural incentives, sociocultural and ethical aspects, needs to be better understood.

\section{Incentives and conflict of interest}

Guiding cancer care through the setting of appropriate incentives for the benefit of patients is an enormous challenge, especially for LMIC countries. An incentive is something that motivates participants, such as individuals, entities or organizations, to perform specific actions. Participants can be grouped according to the different ways in which they become motivated to take a particular way of action. For cancer care physicians, public and professional awareness of incentives is usually limited to financial/remunerative effects and is frequently perceived in a negative manner. It is usually argued that physicians have a moral responsibility to trustfully place the needs and interests of patients ahead of their own, free of unwarranted outside influence on their decisions [1]. Incentives are mainly considered in the context of influencing the immediate personal decisions and behavior of cancer specialists or other cancer care professionals, causing potential $\mathrm{CoI}$ that may result in bias that affects professional judgment [2]. The perception of these CoI by cancer patients is assumed to reduce trust in healthcare [3]. Nevertheless, it is impossible to completely eliminate $\mathrm{CoI}$ from healthcare systems.

Incentives and $\mathrm{CoI}$ are commonly considered from the financial perspective and personal advantages only of the involved physicians [4] whereas other professions are usually out of focus in this context. Various professional organizations have demanded that physicians and their medical judgements should be completely independent of any financial interests that may influence their decisions [4]. The aim of this rigorous and general statement is clear and not disputable, but from the healthcare system perspective it has some shortcomings. Furthermore, CoI are almost exclusively considered as the personal (potential) bias of physicians that may result in harm to patients. Due to the complex treatment of cancer patients, the high usage of resources and high treatment costs, cancer care is considered to be especially vulnerable in this regard. The severity of CoIs has been "quantified" by evaluating their likelihood of biasing professional judgment, and the magnitude/extent of the harm caused [5]. Although these considerations are of high importance for cancer patients' benefits and safety, their restriction to financial aspects and the personal benefits of physicians again has various limitations. These shortcomings and limitations are discussed below.

Physicians are not the only players involved in making cancer care decisions. Various other professions in addition to physicians are directly involved in medical decision making, or provide very influential frameworks for making these decisions at the organizational or health system level. Nurses, pharmacists, medical technicians and others regularly decide about treatment opportunities and medical applications for cancer patients in their own professional environment. For example, in the palliative care of cancer patients with metastatic disease, various non-physician professions have to be involved according to the current medical guidelines. These additional players are also subject to the effects of incentives and CoI.

Other sources of CoI apart from financial interests exist, and their impact also needs to be considered. Recently, bias by academic reputation at the individual and institutional level has gained increasing recognition as a source of CoI. Medical education has also attracted attention as an additional CoI category, especially in LMIC countries. Furthermore, healthcare politicians and other non-medical professions who are involved in the definition and implementation of the healthcare system for a given country or region act within their own framework of interests, which may include re-election, economic priorities and sociocultural aspects. This can influence their decisions independently of the immediate benefit for individual patients. Moreover, even cancer patients and healthy members of a society can gain and induce incentives within the healthcare system (for example via patient organizations) that can be independent of improved health benefits. In addition, incentives related to business models for cancer care are also frequently connected to CoI.

It is a misleading assumption that $\mathrm{CoI}$ can be excluded by correct management. The positive as well as negative effects of business models or legal frameworks for cancer care delivery on decisional pathways and on the behavior of healthcare workers or institutions have been rarely investigated to date, despite their prevalence and influence. The available studies have usually investigated healthcare systems in industrial countries, and have focused on financing systems, reimbursement structures and their effects on inefficiencies caused by over- and cross-referrals, unnecessary diagnostics or overtreatment, etc. Cancer care has been almost completely ignored by these scientific evaluations, and the specific impact on cancer care of advanced and metastatic disease remains to be investigated. Nevertheless, 
it is important to recognize that although the framework of a given cancer care system will induce incentivizing effects that may result in decisional $\mathrm{CoI}$, these $\mathrm{CoI}$ are not necessarily related to potential harm for patients.

\section{The impact of health systems and other frameworks on UHC and cancer care}

A number of key aspects of incentives in healthcare systems are of special importance for LMICs with regard to their approaches for achieving UHC for cancer care. Human beings as well as institutional providers are actors within these healthcare systems. Any type of organized economic, political and legal framework for healthcare will introduce motivations for these actors through incentivizing effects that will drive delivery processes and have an impact on efficiency, effectivity and medical quality. Each participant within cancer care systems, be that physicians and other healthcare workers, managers and administrative staff, or patients, will be influenced in their behavior and personal decision-making by the given framework. Even prioritization of healthcare benefits towards cancer care is frequently affected by incentives that arise from the healthcare framework. For example, the distribution and usage of limited healthcare budgets into cancer care development results from a decision to support specific services, such as prevention versus costly drug treatment versus state-of-the-art palliative care. As a consequence, other delivery processes and other patients will have lower budgetary priority. This cannot be avoided and is not negative per se. However, a major issue is to ensure transparency is this process.

The consequences and incentivizing effects of cancer care delivery and reimbursement structures are highly dynamic, and are themselves dependent on the framework and conditions of the entire healthcare system of a given country. This means that developing LMICs need different approaches to achieve UHC compared to industrial countries, particularly with regard to the incentivizing effects of their framework and healthcare systems. This is the main reason why simple "copy \& paste" of cancer care solutions and processes between countries usually fails to achieve the intended aims. With regard to UHC, most LMICs focus primarily on nationwide equal access, assurance of accessibility and reduction of barriers to healthcare usage, whereas efficiency and quality of care are of higher priority in industrial countries.

Medical decisions that involve cancer patients are always made within the context of an environment that is determined by factors that include the human society, social perceptions and values, the ethical framework, healthcare system characteristics, economics and education levels. The fact that the decisional framework for patient-related issues is determined by factors that are not necessarily directly related to individual cancer patients is also acknowledged by the WHO. Moreover, the conception and implementation of a cancer care framework is a major task of healthcare politics. This is justified in terms of generating the right incentives to guide cancer care delivery within an existing system in the intended manner. In the positive sense, this represents the outworking of political and social priorities in which cancer is an important factor, but not the sole consideration.

\section{Perspective: the challenges ahead}

In view of the considerations outlined above, it is clear that a major goal during the implementation and development of measures in LMICs that foster UHC must be not only to minimize the negative effects of $\mathrm{CoI}$ or incentives for single players in the cancer care system with regard to patient benefit/harm, but also to discriminate within the entire system between

(a) targeted effects (intended incentivizing efforts that are not necessarily positive for each individual cancer patient, but which are positive for the entire society/ cancer patient community),

(b) negative consequences of the framework (which are not directly attributable to individual players within the cancer care system) and

(c) misleading/harmful prioritization of secondary individual interests by participants in the cancer care delivery system (not limited to physicians).

In many countries the balance between targeted effects and negative consequences of the healthcare system framework is a matter of continuous political, ethical and professional debate. The main goals are the improvement of the health of the population, optimization of service efficiency and the stimulation of competition between insurers or health care providers regarding costs and quality of care. In LMICs, public transparency of these discussions especially with regard to cancer care is rather limited.

Implementation and modification of the framework for cancer care to achieve UHC for the entire population is usually accompanied by intensive and rapid changes in the delivery processes and system structure. This includes complex changes in clinical routines, in collaborative patterns among different healthcare providers and disciplines, in the behavior of providers, patients or other stakeholders, and in the organization of cancer care. However, these developments can have severe implications with regard to the abovementioned guiding and incentivizing effects. Even different stages of implementation, such as the roll-out phase of insurance compared to full population coverage, can profoundly impact on incentives and motivation to take specific actions 
both at the individual and at the organizational level. Therefore, incentivizing effects have to be considered as highly dynamic processes within developing cancer care systems, and their impact needs to be used carefully and intentionally.

More broadly, specific research programs are urgently required to provide an evidence-based background that can support the implementation of incentives in cancer care. This will be particularly important for highly vulnerable populations, including cancer patients with advanced stages and metastatic disease.

\section{References}

1. Lichter AS (2017) Conflict of Interest and the integrity of the medical profession. JAMA 317(17):1725-1726. https://doi.org/ 10.1001/jama.2017.3191
2. Stead WW (2017) The complex and multifaceted aspects of conflicts of interest. JAMA 317(17):1765-1767. https://doi.org/10. 1001/jama.2017.3435

3. Armstrong K, Freiberg AA (2017) Challenges and opportunities in disclosing financial interests to patients. JAMA 317(17):17431744. https://doi.org/10.1001/jama.2017.2656

4. Fineberg HV (2017) Conflict of interest: why does it matter? JAMA 317(17):1717-1718. https://doi.org/10.1001/jama.2017. 1869

5. McCoy MS, Emanuel EJ (2017) Why there are no "potential" conflicts of interest. JAMA 317(17):1721-1722. https://doi.org/ 10.1001/jama.2017.2308

Publisher's Note Springer Nature remains neutral with regard to jurisdictional claims in published maps and institutional affiliations. 